\title{
Microbial community structure elucidates performance of Glyceria maxima plant microbial fuel cell
}

\author{
Ruud A. Timmers • Michael Rothballer • \\ David P. B. T. B. Strik • Marion Engel • \\ Stephan Schulz • Michael Schloter • Anton Hartmann • \\ Bert Hamelers · Cees Buisman
}

Received: 18 October 2011 /Revised: 8 January 2012 / Accepted: 10 January 2012 /Published online: 25 February 2012

(C) The Author(s) 2012. This article is published with open access at Springerlink.com

\begin{abstract}
The plant microbial fuel cell (PMFC) is a technology in which living plant roots provide electron donor, via rhizodeposition, to a mixed microbial community to generate electricity in a microbial fuel cell. Analysis and localisation of the microbial community is necessary for gaining insight into the competition for electron donor in a PMFC. This paper characterises the anode-rhizosphere bacterial community of a Glyceria maxima (reed mannagrass) PMFC. Electrochemically active bacteria (EAB) were located on the root surfaces, but they were more abundant colonising the graphite granular electrode. Anaerobic cellulolytic bacteria dominated the area where most of the EAB were found, indicating that the current was probably generated via the hydrolysis of cellulose. Due to the presence of oxygen and nitrate, short-chain fatty acid-utilising denitrifiers were the major competitors for the electron donor. Acetate-utilising
\end{abstract}

Ruud A. Timmers and Michael Rothballer contributed equally to this work.

R. A. Timmers · D. P. B. T. B. Strik · B. Hamelers $(\bowtie) \cdot$

C. Buisman

Sub-department of Environmental Technology,

Wageningen University,

Bornseweilanden 9,

6708 WG Wageningen, the Netherlands

e-mail: bert.hamelers@wur.nl

M. Rothballer $\cdot$ A. Hartmann

Department Microbe-Plant Interactions, Helmholtz Zentrum

München, German Research Center for Environmental Health, Ingolstädter Landstrasse 1,

85764 Neuherberg, Germany

M. Engel $\cdot$ S. Schulz $\cdot$ M. Schloter

Department Terrestrial Ecogenetics, Helmholtz Zentrum

München, German Research Center for Environmental Health, Ingolstädter Landstrasse 1,

85764 Neuherberg, Germany methanogens played a minor role in the competition for electron donor, probably due to the availability of graphite granules as electron acceptors.

Keywords 454 amplicon sequencing · Geobacter . Microbial community · Plant microbial fuel cell · Renewable energy $\cdot$ Rhizosphere

\section{Introduction}

The plant microbial fuel cell (PMFC) is a new technology that can potentially provide renewable and sustainable energy. The PMFC transforms solar energy into electricity through the oxidation of organic compounds originating from photosynthesis (De Schamphelaire et al. 2008; Strik et al. 2008). Electricity generation in the PMFC is based on the loss of organic compounds by plant roots (rhizodeposition) (Pinton and Varanini 2007) and oxidation of these organic compounds by electrochemically active bacteria (EAB) (Potter 1911). In the PMFC, electrons, proton and carbon dioxide are produced by oxidation of organic compounds lost by plant roots in the anode. The electrons that are transferred to the anode electrode are consumed in the cathode compartment by typically reduction of oxygen to water (Strik et al. 2011).

Several bacterial species are known to produce current in microbial fuel cells (MFC): Shewanella putrefaciens using lactate, pyruvate and formate as electron donor (Kim et al. 1999; Park and Kim 2001); Clostridium butyricum and Clostridium beijerinckii, using glucose, starch, lactate and molasses (Niessen et al. 2004; Park et al. 2001); Geobacter sulfurreducens, using acetate and hydrogen (Bond and Lovley 2002); Rhodoferax ferrireducens, using glucose (Chaudhuri and Lovley 2003); Geobacter metallireducens, using acetate (Min et al. 2005) and Rhodopseudomonas palustris, using 
acetate, lactate, valerate, fumarate, ethanol, glycerol and yeast extract (Xing et al. 2008). Alcaligenes faecalis, Enterococcus gallinarum and Pseudomonas aeruginosa are known to generate electricity by producing their own mediators, using glucose as an electron donor (Rabaey et al. 2004). To date, Enterobacter cloacae is the only pure culture that is known to generate electricity directly from more complex electron donors, such as cellulose (Rezaei et al. 2009).

Rhizodeposition is the loss and release of organic and inorganic compounds by plant roots into the rhizosphere (the soil volume affected by the presence of plant roots). Rhizodeposition provides a carbon and energy source, thus stimulating the development of bacterial communities in the rhizosphere. In anaerobic environments, rhizodeposition can also provide oxygen as an electron acceptor and may stimulate the development of facultative anaerobic bacterial communities (Hartmann et al. 2009). In addition to prokaryotes (bacteria and archaea), eukaryotes (such as fungi, protozoa, nematodes and meso- and macro-fauna) are also found in the rhizosphere (Phillips et al. 2003).

The genera Geobacter and Desulfobulbus have been found in PMFCs (De Schamphelaire et al. 2010). As both genera contain electrochemically active species that are enriched at the anode of MFCs (Holmes et al. 2004; Jung and Regan 2007), it can be assumed that EAB may have been present in the PMFC. However, no studies have yet confirmed the presence of active EAB in PMFCs. The presence of active $\mathrm{EAB}$ is required to prove their role in electricity production by PMFCs.

In a PMFC, the plant roots are located at the anode of the MFC, where rhizodeposition provides $\mathrm{EAB}$ with electron donor. Together the MFC anode and the rhizosphere make up a complex system. In this complex system, it is likely that different microorganisms compete for electron donor. Competition for electron donor among the different microorganisms results in a decrease of electron donor available for EAB and thus lower current generation. Analysis and localisation of the microbial community is needed to gain insight into the competition for electron donor in a PMFC.

In this study, we determined the microbial communities present in a PMFC rhizosphere and localised the active EAB in the rhizosphere and on the electrode of the PMFC. Additionally, we investigated the electron donor and electron pathways in the PMFC. We used 454 technology to sequence 16S rRNA gene amplicon libraries from bacteria and archaea. To prove the relevance of dominant pylogenetic groups and to localise those bacteria and archaea, fluorescent in situ hybridisation (FISH) in combination with confocal laser scanning microscopy (CLSM) was performed in samples of high-current-generating and low-currentgenerating Glyceria maxima (reed mannagrass) PMFC.

\section{Materials and methods}

Preparation of PMFC

The anode compartment of the PMFC consisted of $165 \mathrm{~g}$ of graphite granules with diameters between 1 and $2 \mathrm{~mm}$ (le Carbone, Wemmel, Belgium). The graphite granules were placed in a glass cylinder (Schott, Duran) with a diameter of $0.035 \mathrm{~m}$ and a height of $0.30 \mathrm{~m}$. To separate the anode from the cathode, a cation exchange membrane (fumasep ${ }^{\circledR}, \mathrm{FKB}$, Fumatech $\mathrm{GmbH}$, St. Ingbert, Germany) was fixed at the bottom of the glass cylinder. The anode was placed in the cathode, which consisted of a PVC beaker with a diameter of $0.11 \mathrm{~m}$ and a height $0.04 \mathrm{~m}$ that contained a $6-\mathrm{mm}$-thick graphite felt (Coidan Graphite Products LTD, York, UK). Figure 1 shows a schematic representation of the used setup.

To close the electrical circuit, two 4-cm golden wires glued to Teflon coated copper wire were connected over a resistance of $900 \Omega$. To create contact between the anode and the cathode, one of the gold wires was placed in between the graphite granules and the other was woven through the graphite felt.

\section{Preparation of graphite granules}

Eight setups were operated without plants to develop electrochemically active biofilms on the graphite granules. The anode was inoculated with $5 \mathrm{ml}$ of anolyte from an MFC running on acetate (Dekker et al. 2009), and the anolyte was extracted with a syringe. The setups were operated in batch mode. As nutrient solution 1/2 Hoagland Medium (Taiz and Zeiger 2006) buffered with an 8-mM potassium phosphate buffer solution ( $\mathrm{pH}$ 6.8) was used. The electron donor was

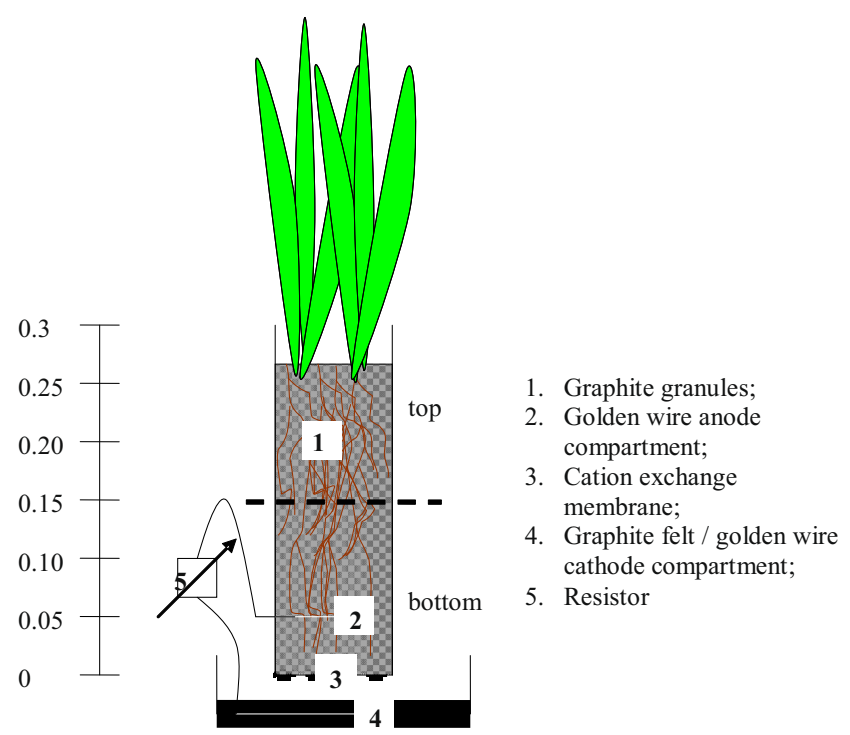

Fig. 1 Schematic representation of the PMFC setup and location of the samples 
supplied by adding $10 \mathrm{mM}$ potassium acetate to the nutrient solution of setups 1 through 4 and $10 \mathrm{mM}$ glucose to the nutrient solution of 5 through 8 . After 40 days, the acetate-fed setups generated a current density of $0.248 \pm 0.004 \mathrm{Am}^{-2}$, and the glucose fed setups generated a current density of $0.151 \pm$ $0.02 \mathrm{Am}^{-2}$. Current densities are expressed per square meter of cross-sectional area of the PMFC $\left(0.00096 \mathrm{~m}^{2}\right)$ throughout the document.

Before the graphite granules were used for the six PMFCs, they were thoroughly mixed to ensure the presence of microorganisms able to generate electricity from acetate as well as glucose. After the mixing, the graphite granules were rinsed with tap water to remove a large part of the residual electron donor. One stem of G. maxima (D'n Bart Waterplanten, Handel, the Netherlands) with a weight between 4.4 and $7.8 \mathrm{~g}$ was planted in each PMFC.

\section{Operation of PMFCs}

The medium used to feed the PMFCs was $1 / 2$ Hoagland buffered with $0.008 \mathrm{~mol}^{-1}$ potassium phosphate buffer solution as described by Timmers et al. (2010) ( $\mathrm{pH} \mathrm{6.8,}$ conductivity between 1.5 and $1.7 \mathrm{mS} \mathrm{m}^{-1}$ ). The medium was fed through a sample point located $0.07 \mathrm{~m}$ above the graphite granules. The applied flow rate was $0.17 \mathrm{ml} \mathrm{s}^{-1}$ throughout the experimental period. The feeding frequency of the PMFC with buffered Hoagland medium was $5 \mathrm{~min}$ every $12 \mathrm{~h}$.

Both MFC and PMFC setups were placed in a climate control cabinet (Microclima 1750 Snijders, Tilburg, the Netherlands). In this manner, environmental conditions were fixed at illumination period of $14 \mathrm{hday}^{-1}$, average light density in the photo active region of $596 \pm 161 \mu \mathrm{mol} \mathrm{m}{ }^{-2} \mathrm{~s}^{-1}$, temperature of $25^{\circ} \mathrm{C}$ and humidity of $75 \%$.

Sample selection, preparation and DNA extraction

Samples for analysis were taken from PMFC generating the highest current (PMFC6) and the PMFC generating the lowest current (PMFC3). Both PMFCs were dismantled 225 days after the start of the experiment. The anode compartments of both PMFCs were divided into bottom ( 0 to $0.15 \mathrm{~m})$ and top $(0.15$ to 0.3$)$ sections. Figure 1 shows a schematic representation of the PMFC and the sampling points. The root samples were taken from all of the sampling points in both PMFCs and stored at $-80^{\circ} \mathrm{C}$. DNA was extracted from a total of $500 \mathrm{mg}$ of root material per sample following the protocol of Griffiths et al. (2000) and using a BIO101 lysing matrix (MP Biomedicals, Illkirch, France) and a final purification step with the AllPrep Mini Kit by Qiagen (Munich, Germany). The amount of DNA in solution was quantified using a bioanalyser from Agilent (Böblingen, Germany).
PCR, 454 sequencing and data processing

If not otherwise mentioned, all the steps were performed according to the Roche 454 sequencing protocol for amplicons. To generate the amplicon library for bacteria and archaea, specific primers were selected according to two criteria: (a) the fragment should span 600 bases of the $16 \mathrm{~S}$ rRNA gene to receive sufficient phylogenetic information and (b) the primers should bind to as many bacterial/archaeal sequences as possible without detecting non-target groups. To verify these criteria, the ARB probe match tool was used with the latest SILVA database (containing over 400,000 sequences), resulting in the following bacterial $16 \mathrm{~S}$ primers: 926-F (5'-AAACTYAAAKGAATTGACGG-3', Escherichia coli position 907-926 (Lane 1991)) and 630R (5'-CAKAAAGGAGGTGATCC-3', E. coli position 1528-1544 (Juretschko et al. 1998)). The archaeal primers were rSAf(i) (5'-CCTAYGGGGCGCAGCAG-3', E. coli position 341-357 (Nicol et al. 2003)) and 958r (5'YCCGGCGTTGAMTCCAATT-3', E. coli position 940958 (Bano et al. 2004)). These primers were extended as amplicon fusion primers with respective A and B adapters, key sequence and multiplex identifiers (MID) as recommended and tested in initial PCR reactions to determine their optimal annealing temperatures $\left(50^{\circ} \mathrm{C}, 54^{\circ} \mathrm{C}, 58^{\circ} \mathrm{C}\right)$, cycle numbers $(20,22,25,30)$ and amounts of template DNA (50, $100,200 \mathrm{ng})$. The conditions under which a sufficient amount (approximately $10^{12}$ molecules) of specific amplicons of the right size was generated using the lowest number of cycles in all the samples were determined $\left(50^{\circ} \mathrm{C}\right.$, 22 cycles, $50 \mathrm{ng}$ ) for bacterial $16 \mathrm{~S}$ rRNA genes. For archaeal $16 \mathrm{~S}$ rRNA genes, 30 cycles and the addition of $8 \%$ DMSO were necessary to obtain sufficient PCR product for archaea. These conditions were then used to produce four amplicon libraries each (top and middle/bottom part of the high- and low-current-producing PMFC). The PCR products were purified with AMPure Beads (Agencourt, Beckman Coulter, Krefeld, Germany) and pooled in equimolar amounts.

Emulsion PCR, emulsion breaking of DNA-enriched beads and sequencing run of the amplicon pools were performed on a second-generation pyrosequencer (454 GS FLX Titanium, Roche) using titanium reagents and titanium procedures as recommended by the developer following protocols for bidirectional amplicon sequencing. Quality filtering of the pyrosequencing reads was performed using the automatic amplicon pipeline of the GS Run Processor (Roche) to remove failed and low-quality reads from raw data and to remove adaptor sequences.

Newbler assembler v 2.3 (Roche) was used to batch sequences based on MID-identifiers and to combine corresponding sequences derived from forward and reverse reads with a similarity of $99 \%$ and an overlap of 400 bases for the bacterial 
sequences. Due to the low sequence diversity, a similarity value of $98 \%$ and an overlap of 200 bases were sufficient for archaeal sequences. The consensus sequences were inspected for chimera with the help of the Bellerophon software (http://foo. maths.uq.edu.au/ huber/bellerophon.pl) and putative chimera were removed from the dataset.

The alignment and phylogenetic analyses were performed using the ARB 5.1 software package (Strunk and Ludwig 1997). After phylogenetic allocation of the sequences down to the family or genus level, the sequences belonging to different phylogenetic groups were added together and depicted as a percentage of the total sequencing reads obtained from each sample. Phyla represented by less than 50 sequencing reads in all four libraries totalled were not included. The assembled unique sequences with their phylogenetic allocation were deposited under the numbers JF731380 and JF732737 in GenBank.

Quantitative real-time PCR of archaeal and bacterial 16S rRNA genes

For Sybr ${ }^{\circledR}$ Green-based quantitative real-time PCR (qRT-PCR), the same primers as for the 454 sequencing were used, but without the 454 specific adaptor, the key and the MID sequences. For standard generation, PCRs using these specific primers were performed with the same conditions as for the amplicon library amplification. The resulting products were cloned into a pSC-AAmp/Kan Vector using the StrataCloneTM PCR Cloning Kit (Agilent, Palo Alto, CA, USA) after manufacturer's instructions. In each case, eight resulting clones were picked, the inserts sequenced and subsequently allocated by the ARB software package. Sequences either related to the genus Methanobacterium (archaea) or to the genus Clostridium (bacteria) were chosen as standards, as both sequences represented genera commonly found in a large proportion in all four amplicon libraries.

Subsequently, abundances of archaeal and bacterial 16S rRNA genes were measured on the ABI Prism 3300 system (Applied Biosystems, Foster City, CA, USA) under comparable conditions $\left(10 \mathrm{~min} 95^{\circ} \mathrm{C}, 40\right.$ cycles of $20 \mathrm{~s} 95^{\circ} \mathrm{C}, 1 \mathrm{~min}$ $50^{\circ} \mathrm{C}$ and $30 \mathrm{~s} 72^{\circ} \mathrm{C}$ ) in triplicates. Signal acquisition was done at $78^{\circ} \mathrm{C}$ to overcome bias due to primer dimers. PCR reaction mixtures ( $25 \mu \mathrm{l}$ volume) contained $2 \mathrm{mM} \mathrm{MgCl}$, $0.1 \mu \mathrm{M}$ of respective primers (forward and reverse), $1 \times$ Power Sybr ${ }^{\circledR}$ Green (Applied Biosystems, Foster City, CA, USA) and 4 ng of template DNA. Curves for both standards were linear $\left(r^{2}>0.99\right)$ over five orders of magnitude, and amplification efficiencies were comparable at $87 \%$.

Fluorescent in situ hybridisation and confocal laser scanning microscopy

Fixed bacterial samples were stained for $10 \mathrm{~min}$ in the dark with the DNA binding dye Syto Orange 80 (Molecular
Probes, Invitrogen, Carlsbad, CA, USA) at a concentration of $5 \mu \mathrm{M}$ in $10 \mathrm{mM}$ Tris, $1 \mathrm{mM}$ EDTA $(\mathrm{pH} \mathrm{8.0)}$ and subsequently washed twice with ultrapure water. For the FISH, the washed root samples were fixed for $2 \mathrm{~h}$ at room temperature (Amann et al. 1990) with either 50\% ethanol in ultrapure water for Gram-positive bacteria or $4 \%$ paraformaldehyde for Gram-negative bacteria. Hybridisation with fluorochrome (Cy3, Cy5)-labelled oligonucleotide probes was carried out following the protocols described by Manz et al. (1992) and Amann et al. (1992). For the microscopic observations, the FISH stained root pieces (ca. 5-10 mm long) were embedded in Citifluor (Citifluor Ltd., Canterbury, UK) and observed by CLSM without further cutting.

rRNA-targeted oligonucleotide probes were synthesised and labelled by Sigma-Aldrich (Steinheim, Germany). EUB338-I (Amann et al. 1990) and EUB338-II and EUB338-III (Daims et al. 1999) were used in an equimolar mixture at different formamide concentrations. To detect the genus Geobacter, an equimolar mixture of the 16S rRNA targeted probes Geo1A and Geo1B was used at a $35 \%$ formamide concentration. Geo1A is specific for G. sulfurreducens, Geobacter hydrogenophilus, Geobacter grbiciae and G. metallireducens; Geo1B detects most other Geobacter species (Demaneche et al. 2008). To detect the Ruminococcaceae group members Ruminococcus bromii, Clostridium sporosphaeroides and Clostridium leptum, the Rbro730 probe (Harmsen et al. 2002) was applied in the presence of $20 \%$ formamide. To detect most members of Clostridium clusters I and II, the Chis150 probe (Collins et al. 1994; Franks et al. 1998) was used with $35 \%$ formamide.

The fluorescently labelled cells were detected using a Zeiss LSM510 CLSM. An argon ion laser supplied 488-nm illumination to excite the fluorescein, and two helium-neon lasers provided $543 \mathrm{~nm}$ for Cy 3 and $633 \mathrm{~nm}$ for Cy5. For each hybridisation probe, an EUB338 mix labelled with Cy5 (blue) was combined with another group-specific probe labelled with $\mathrm{Cy} 3$ (red). The binding of both probes resulted in a magenta staining of the target cells. The green channel (fluorescein) served as a negative control that showed only autofluorescence by the root or other particles. Syto Orange was detected by excitation with the 488- and 543-nm laser wavelengths, resulting in a yellow/orange fluorescence signal in the target cells; the negative control was the blue channel (excitation at $633 \mathrm{~nm}$ ).

The optical sectioning of the root was achieved by moving the focus position deeper into the sample in $1 \mu \mathrm{m}$ steps, which produced $\mathrm{z}$-stacks of individual pictures from the same $x y$-area with a penetration depth of up to $60 \mu \mathrm{m}$. The resulting set of pictures was displayed as an orthogonal view using the LSM510 software package that was provided by Zeiss. 


\section{Results}

\section{Energetic performance of PMFCs}

The daily average current density was $0.067 \pm 0.058 \mathrm{Am}^{-2}$ for the low-current PMFC and $0.164 \pm 0.065 \mathrm{Am}^{-2}$ for the highcurrent PMFC (Fig. 2). The daily average current density was substantially higher than the $0.009 \mathrm{Am}^{-2}$ reported for freshwater sediment microbial fuel cells (Holmes et al. 2004). Furthermore, the daily average current density was in the range of average current densities reported in literature for PMFCs (De Schamphelaire et al. 2008; Kaku et al. 2008; Strik et al. 2008; Timmers et al. 2010; Helder et al. 2010). An examination of the standard deviations revealed that both PMFCs had relatively large fluctuations in current density over time. The maximum power density, with an external resistance of $900 \Omega$, was $32 \mathrm{~mW} \mathrm{~m}^{-2}$ for the low-current PMFC and $80 \mathrm{~mW} \mathrm{~m}^{-2}$ for the high-current PMFC. The high-current PMFC produced a total of $522 \mathrm{~J}$, and the lowcurrent PMFC produced a total of $124 \mathrm{~J}$.

Differences in the 454 sequencing of bacterial and archaeal 16S rRNA gene fragments between PMFC

A bidirectional sequencing approach of the four pooled bacterial 16S rRNA gene amplicon libraries (top section low-current PMFC, middle/bottom section low-current PMFC, top section high-current PMFC, middle/bottom section high-current PMFC) resulted in a total of 140,000 reads with 114,000 key pass reads and 46,000 reads that passed all of the internal quality filters of the 454 software. An average read length of 517 bases and almost no reads shorter than 500 bases were obtained. The sequencing of the bacterial amplicon libraries was repeated once for verification of the results with slightly lower output and read lengths but no major differences in bacterial abundance.

The assembly resulted in 495 unique sequences for bacteria and 863 for archaea, which were then phylogenetically allocated by the ARB software package. Figure 3a shows the

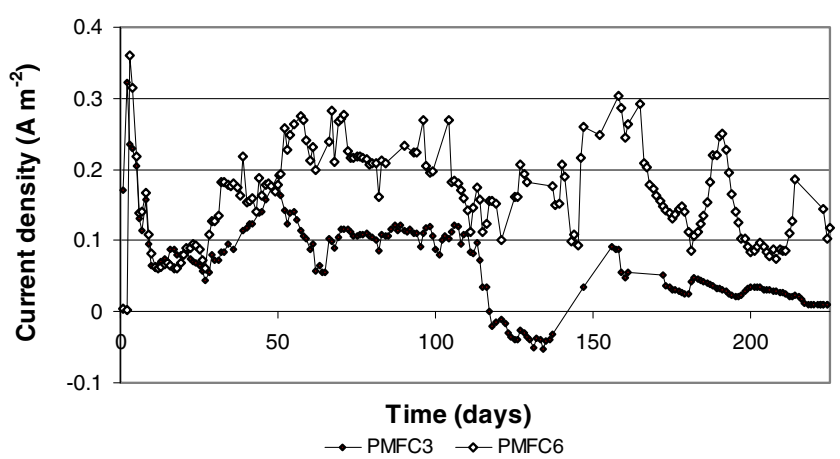

Fig. 2 Current density versus time of the low-current-density PMFC (PMFC3) and high-current-density PMFC (PMFC6) bacterial classes and families found in both PMFCs. In the bottom section of the high-current PMFC, the major portion (54\%) of the bacterial $16 \mathrm{~S}$ rDNA belonged to the Ruminococcaceae family, with considerable amounts $(25 \%)$ belonging to the Clostridiaceae family. Within the Ruminococcaceae family, almost all the sequences clustered around C. sporosphaeroides. Although unambiguous species-level assignment is difficult using the 16S rDNA fragment lengths of $600 \mathrm{bp}$ that were obtained, this analysis enabled the selection of suitable FISH probes for verification of the sequencing results.

In the top section of the high-current PMFC, the major portion $(36 \%)$ of the bacterial $16 \mathrm{~S}$ belonged to Comamonadaceae, and considerable amounts could be assigned to the Rhodocyclaceae (10\%) and Clostridiaceae families (13\%). The bottom and top sections of the low-current PMFC were dominated (54\% and $35 \%$, respectively) by members of the Comamonadaceae family, but also representatives of the Rhodocyclaceae family were frequently detected (13\% and $23 \%$ ).

Figure $3 \mathrm{~b}$ shows the archaeal genera found in both PMFCs. In the high-current PMFC, the genus Methanobacterium made up over $95 \%$ of the archaeal community. In the low-current PMFC, the archaeal community was more diverse and mainly consisted of the genus Methanobacterium and uncultured Methanobacteriaceae. Furthermore, Methanosarcina was found to account for $5 \%$ of the archaeal community in the top section and up to $10 \%$ in the bottom section of the low-current PMFC.

The quantification of archaeal and bacterial 16S rRNA genes revealed great differences in the abundances of about two to three orders of magnitude. Gene copy numbers of bacterial 16S rRNA genes per nanogram extracted DNA were (top section, low current; middle/bottom section, low current; top section, high current; middle/bottom section, high current) $0.9,2.4,0.7$ and $1.2 \times 10^{6}$, respectively. In comparison, gene copy numbers of archaeal $16 \mathrm{~S}$ rRNA were $5.6,4.9,1.4$ and $0.8 \times 10^{3}$, respectively. While the abundances of bacteria differed by a maximum of factor 2, when comparing the same sections of the high and the low-current PMFCs, there were four to six times more archaeal $16 \mathrm{~S}$ genes in the low-current than in the high-current PMFC.

Localisation of characteristic PMFC bacteria by FISH and CLSM

A biofilm was detected, particularly in roots from the upper sections of both PMFCs, where the film completely surrounded the root and was 5 to $10 \mu \mathrm{m}$ thick. No visual differences in the structure or amount of the biofilms were observed between the PMFCs. For the low-current PMFC, however, fungal hyphae were clearly detected in the biofilms of all observed samples.

FISH was used to localise Geobacter, Clostridiaceae and Ruminococcaceae species that were previously detected by 
a

Bacterial families

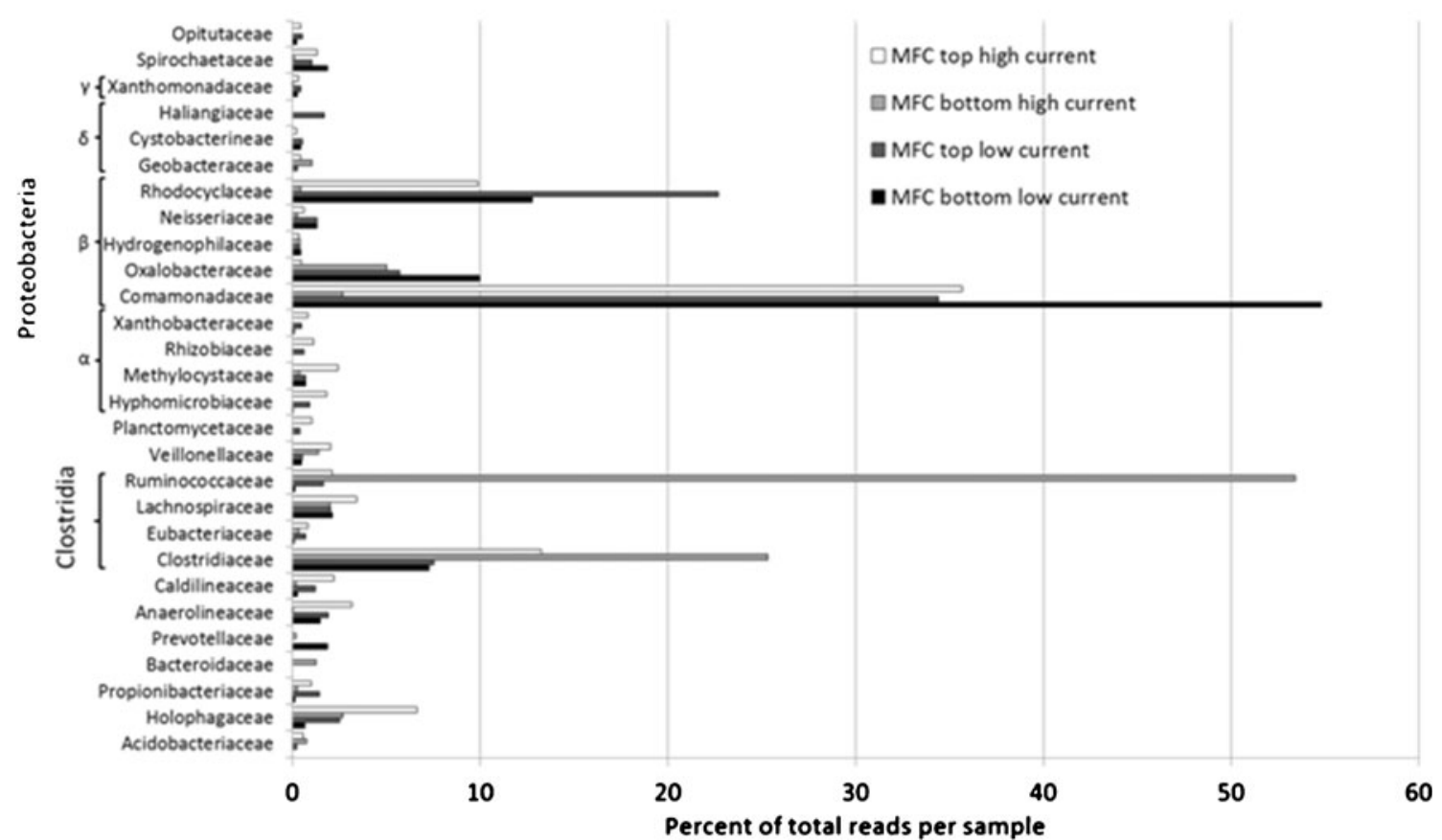

b

Archaeal genera

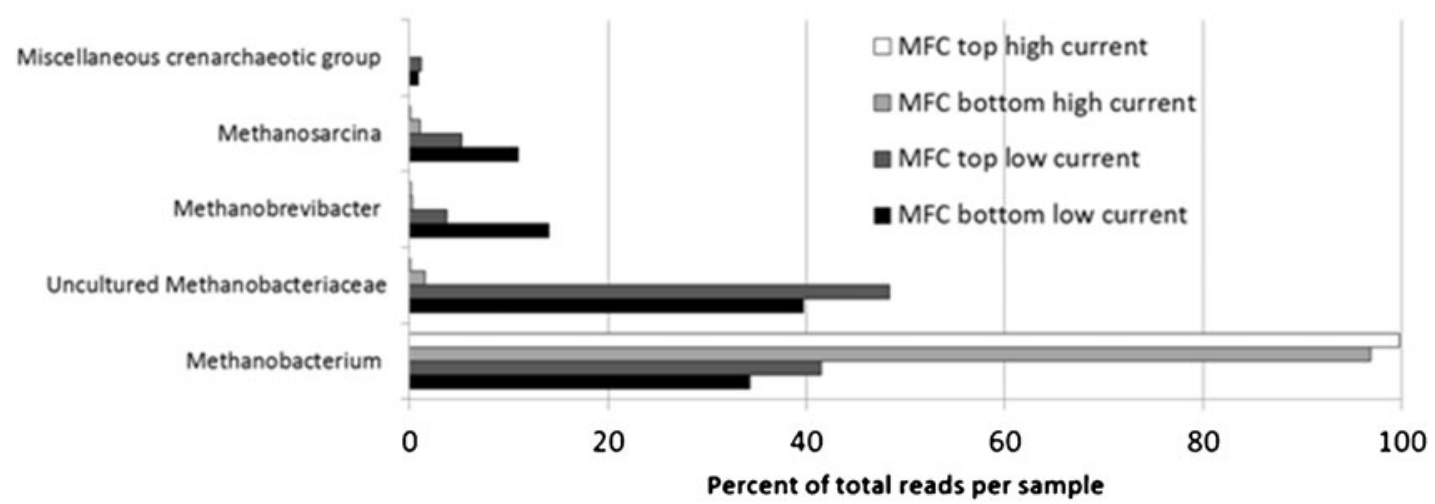

Fig. 3454 sequencing reads in percent of total reads per sample phylogenetically allocated to different families/genera by the ARB software package. Phyla represented by less than 50 sequencing reads in all four libraries totalled are not included. a Bacteria. b Archaea

454 sequencing of the $16 \mathrm{~S}$ rRNA gene. The Geobacter probe set enabled detection of fluorescent Geobacter cells, which appeared more frequently on the graphite granules than on the root surfaces. With probe Geo1A, specific only for $G$. sulfurreducens, G. metallireducens, G. grbiciae and G. hydrogenophilus, positive signals were identified on both roots and granules from both PMFCs. Furthermore, the positive signal was more frequently observed on samples from the bottom layer of the high-current PMFC. A Clostridia cluster I and II specific probe yielded very few positive signals, and no clear differences were observed between the individual samples. With probe Rbro730, specific for the three Ruminococcaceae species R. bromii, $C$. sporosphaeroides and C. leptum, small numbers of cells were positively identified on the root surfaces, but fluorescent signals were detected to a much greater extent in the outer cortex layers of roots of the high-current PMFC.

\section{Discussion}

The presence of electrochemically active bacteria in the PMFCs

The current generation was likely due to the presence of the active G. sulfurreducens and G. metallireducens that were 
detected in both the PMFCs (Fig. 4c). Pure cultures of both $G$. sulfurreducens and $G$. metallireducens are known to generate electricity in an MFC (Bond and Lovley 2002; Min et al. 2005). In particular, G. sulfurreducens has been shown to colonise the anodes of MFCs in structured biofilms (Holmes et al. 2004; Jung and Regan 2007; Reguera et al. 2006). The same probe also detected active $G$. grbiciae and G. hydrogenophilus. These species have not been shown to be capable of generating electricity in an MFC, but they are able to reduce Fe(III) (Lovley et al. 2000), a property that is also exhibited by EAB such as $S$. putrefaciens, G. sulfurreducens, G. metallireducens (Coates et al. 2001) and R. ferrireducens (Finneran et al. 2003).

In the bottom section of the high-current PMFC, $G$. sulfurreducens or one of its close relatives ( $G$. metallireducens, G. grbiciae and G. hydrogenophilus) were observed frequently on the graphite granules. The roots in the bottom section of the high-current PMFC were also colonised, although less frequently than the graphite granules (Fig. 4c, f). In the low-current PMFC, G. sulfurreducens or one of its close relatives ( $G$. metallireducens, G. grbiciae and $G$. hydrogenophilus) were observed less frequently in the top section, and no signal at all was detected in the bottom section.
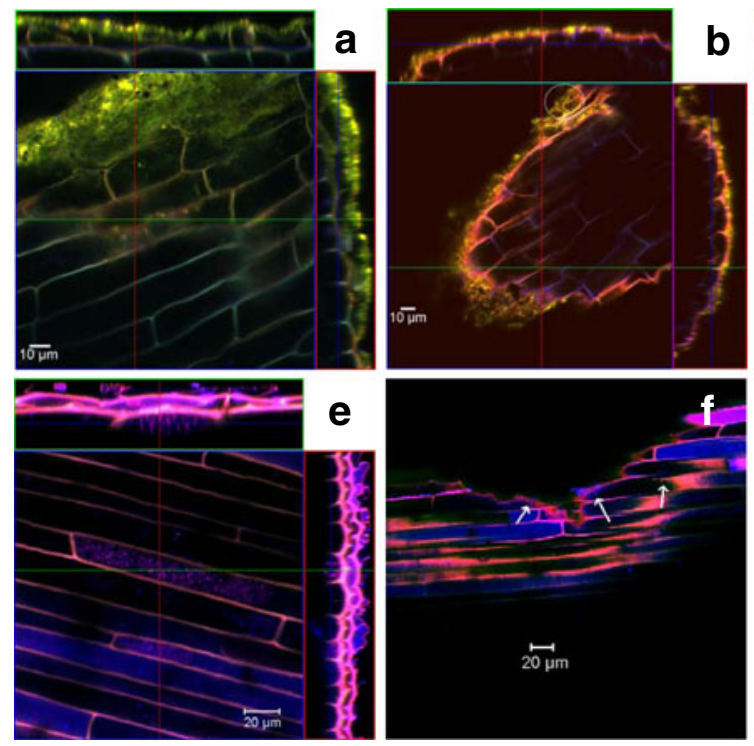

Fig. 4 CLSM images of FISH or SYTO orange stained roots from the two PMFCs. All FISH staining was performed with probe Eub338Mix labeled in $\mathrm{Cy} 5$ (blue) in combination with a group specific probe labelled in Cy3 (red). Target cells appear in magenta (combination of red and blue). If three dimensional z-stacks were prepared, pictures are shown in orthogonal views. The top view, framed in blue, gives one picture from the middle of this z-stack. The red and green lines represent vertical optical cuts through the stack, which result in the side view images framed in red and green, respectively. In these side views, the blue line marks the vertical position, where the top view image is located within the z-stack. a Syto orange stained biofilm on a root from high-current-producing PMFC, upper part. b Syto orange stained biofilm on a root from low-current-producing PMFC, upper
The difference in the presence of the genus Geobacter between the PMFC types was supported by the differences in the presence of the Geobacteraceae family to which the Geobacter genus belongs (Fig. 4a). An average of $0.74 \%$ of the obtained 16S rRNA gene fragment sequences belonged to Geobacteraceae in the high-current PMFC, while an average of only $0.13 \%$ belonged to Geobacteraceae in the low-current PMFC.

Anaerobic Ruminococcaceae were predominant in the bottom section of the high-current PMFC

Ruminococcaceae accounted for over half of the bacterial rhizosphere sequences in the bottom section of the highcurrent PMFC. The members of this family have not been reported to be electrochemically active, but they are known to hydrolyse cellulose into hydrogen and low molecular weight organic compounds. In MFCs, the hydrolysis of cellulose generates electron donors that are suitable for $G$. sulfurreducens (Maki et al. 2009; Ren et al. 2007).

Based on their cellulolytic activity, the Ruminococcaceae species probably played an important role in electricity generation in the PMFC. They probably hydrolysed the cellulose, originating from dead roots, into suitable electron
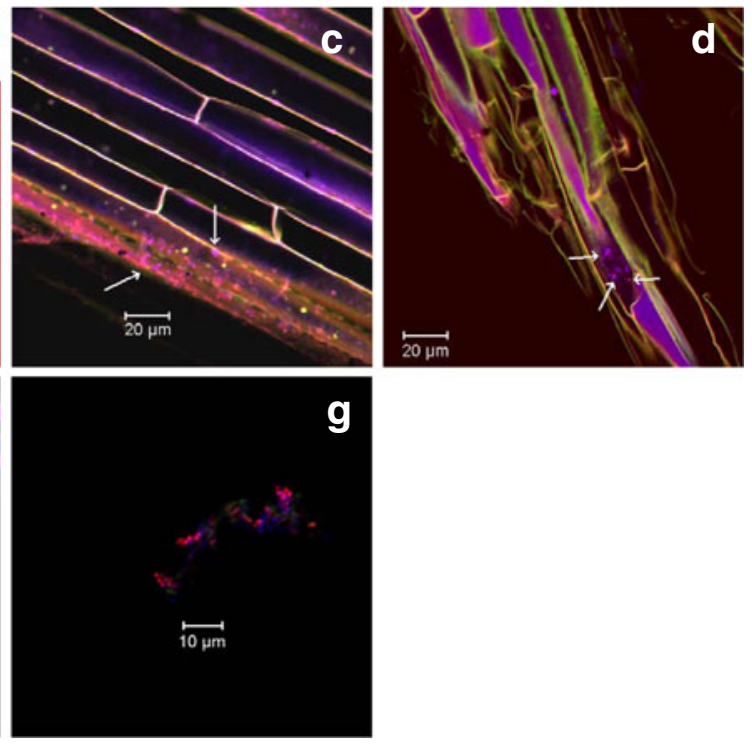

g

part; fungal hyphae are marked by white circle. c FISH stained root with probe set Geo1A and Geo1B (Geobacter genus) from highcurrent PMFC, bottom part (indicated by white arrows). d Root from same sample as 4C; Geo1A (detecting only G. sulfurreducens, $G$. metallireducens, G. grbiciae or G. hydrogenophilus, indicated by white arrows). e FISH stained root with probe Rbro730 (C. sporosphaeroides, C. leptum, R. bromii) from high-current PMFC, middle part. f FISH staining with probe set Geo1A and Geo1B (Geobacter genus) of a graphite granule of high-current PMFC (not visible); small cluster of Geobacter is detected on the surface of the graphite granules (indicated by white arrows). g CLSM picture of a FISH stained root from highcurrent PMFC, bottom part; probe Chis150 (Clostridiaceae) 
donors for EAB. Typically, 35-50\% of dry-plant weight consists of cellulose (Lynd et al. 1999), which stresses the importance of cellulolytic activity in generating a suitable electron donor for EAB.

The main species of the Ruminococcaceae family was probably C. sporosphaeroides, which is able to ferment glutamate to ammonia, $\mathrm{CO}_{2}$, acetate, butyrate and hydrogen via the hydroxyglutamate pathway (Hsiao et al. 2009). Furthermore, this species has been used to improve the hydrolysis rates of cellulose and hemicelluloses in a biomass fermenter (patent no. WO/2010/072219).

C. sporosphaeroides was mainly found in the root cells of the outer root cortex in the high-current PMFC (see Fig. 4e). Due to the large surface area of the outer root cortex, the presence of $C$. sporosphaeroides there probably resulted in a high rate of hydrolysis (Lynd et al. 2002). It could not be determined whether $C$. sporosphaeroides acted as a moderate pathogen and actively killed root cells or was simply colonising dead plant material. E. cloacae, able to generate current from complex electron donors as a pure culture, was not detected in the PMFCs (Rezaei et al. 2009). This is another indication that current was likely generated via the hydrolysis of complex electron donors into suitable electron donor for G. sulfurreducens. The importance of the hydrolysis of cellulose for electricity generation is supported by the results of Ren et al. (2007). They demonstrated that a binary culture of Clostridium cellulolyticum and G. sulfurreducens (with carboxymethyl cellulose and MN301 cellulose as electron donors) produced current, while single cultures of these bacteria did not.

The lack of dominance by obligate anaerobes in the top section of the high-current PMFC and in the top and bottom sections of the low-current PMFC indicates the presence of oxygen. Therefore, hydrolysis in the low-current PMFC was likely performed by fungi and/or facultative anaerobic cellulolytic bacteria, such as the Rhodocyclaceae family (Schellenberger et al. 2010). In contrast, the bottom section of the high-current PMFC was dominated by the obligate anaerobe Ruminococcaceae, indicating that cellulose hydrolysis was predominantly carried out by members of this family.

Short-chain fatty acid-utilising denitrifiers are major competitors for electron donor

Except for the bottom section of the high-current MFC, Rhodocyclaceae and Comamonadaceae were the dominant families observed on the PMFCs. Rhodocyclaceae and Comamonadaceae are known to be facultative anaerobic short-chain fatty acid-utilising denitrifiers (Ginige et al. 2005; Nielsen et al. 2006; Thomsen et al. 2007). Nevertheless, both Comamonadaceae and Rhodocyclaceae have been previously observed in MFCs. Comamonadaceae have been found to dominate the bacterial community in the anode of a cellulose-fed MFC (Rismani-Yazdi et al. 2007). Rhodocyclaceae have been found to dominate the bacterial community in the anode of an acetate-fed MFC with an open air cathode (Borole et al. 2009). Furthermore, Comamonas denitrificans and R. ferrireducens species belonging to the Comamonadaceae family are able to generate electricity in an MFC in the absence of oxygen and nitrate (Xing et al. 2008, 2010).

In our PMFC, both oxygen and nitrate were probably present due to the diffusion of oxygen through the roots and the addition of nitrate through the medium. The nitrate flux into the anode was equivalent to $140 \mathrm{gN} \mathrm{m}^{-2}$ day $^{-1}(2 \times$ $10^{-3} \mathrm{~mol}_{\text {nitrate }} \mathrm{day}^{-1}$, assuming that there was no medium overflow), which is about 125 times higher than the maximum reported rate of nitrogen uptake by emergent macrophytes in a constructed wetland containing G. maxima (Tanner 1996). Therefore, a large portion of the added nitrate was denitrified by facultative anaerobic activity as long as short-chain fatty acids were available as electron donors. Short-chain fatty acids were probably generated by the aerobic cellulolytic activity of Oxalobacteraceae (Lynd et al. 2002) and the anaerobic cellulolytic activity of Rhodocyclaceae (Schellenberger et al. 2010). Offre et al. (2007) associated the presence of Oxalobacteraceae and Comamonadaceae with mycorrhizal roots and suggested a possible symbiosis between both bacterial families and fungi. The presence of fungi in the outer cortex of the roots in the lowcurrent PMFC was consistent with these findings.

The presence of Rhodocyclaceae and Comamonadaceae probably had a negative effect on current generation, as these families do not contribute to electricity generation in the presence of oxygen and nitrate. Furthermore, these families consume short-chain fatty acids, which decreases the amount of short chain fatty acids available for current generation and thus negatively affect current generation. The difference in current generation between the high- and lowcurrent-generating PMFC could likely be attributed to difference in presence of Rhodocyclaceae and Comamonandaceae in the bottom part of both PMFC. In the bottom part of the high-current PMFC, Rhodocyclaceae and Comamonandaceae were almost absent while they were more abundant in the bottom part of the low-current PMFC. As these families consume short-chain fatty acids, it is likely that their presence resulted in lower current generation because less substrate is available for EAB.

The absence of fungi in the outer cortex of roots in the bottom section of the high-current PMFC, together with possible nitrate depletion may have allowed strictly anaerobic cellulolytic microorganisms (Clostridiaceae and Ruminococcaceae) and EAB, such as G. sulfurreducens and $G$. metallireducens, to proliferate. 
Methanogenesis plays a minor role in competition for electron donor

Methanogens can compete with G. sulfurreducens and $G$. metallireducens for electron donors, as they are able to use acetate, formate, methanol, methylamine, $\mathrm{H}_{2}$ and $\mathrm{CO}_{2}$ (Ishii et al. 2008; Thauer et al. 2008; Thomsen et al. 2007). Quantitative real-time PCR amplification with bacterial and archaeal primers using the same DNA template indicated that considerably less of the DNA present in the sample was derived from archaea than from bacteria. The higher abundance of bacterial DNA compared to archaeal DNA was probably due to the presence of alternative acceptors in the medium, such as oxygen, nitrate and sulphate. As long as these acceptors are present, methanogenic archaea are outcompeted by aerobic bacteria, denitrifiers and/or sulphate-reducing bacteria (Scheid et al. 2004; Thauer et al. 2008). Timmers et al. (2011) did not measure acetate concentrations above $2 \mathrm{mg} \mathrm{l}^{-1}$ (the limit of detection) in the PMFCs of which the biological community was analysed. This finding indicated that hydrolysis is the ratelimiting step in that type of cell, assuming that electricity in the PMFC is generated via fermentation of hydrolyses products to acetate and the subsequent oxidation of acetate by G. sulfurreducens and G. metallireducens. The majority of the methanogenic archaeal community, Methanobacteriacea, are unable to utilise acetate (Thauer et al. 2008). And therefore do not compete with G. sulfurreducens and G. metallireducens for acetate. This restriction is supported by the finding that of all the archaeal genera found in both PMFCs, only the Methanosarcina genera belong to acetate-utilising methanogens (Thauer et al. 2008). Methanosarcina comprised, on average, less than $10 \%$ of the archaeal DNA in the low-current PMFC and were barely observed in the high-current PMFC. The relative higher abundance of Methanosarcina in the low-current PMFC compared to the high-current PMFC likely contributed to competition for acetate. The competition for acetate between $G$. sulfurreducens and G. metallireducens and Methanosarcina likely contributed to the difference in current generation, however less than Rhodocyclaceae and Comamonandaceae. Furthermore, Methanosarcina is a methanogen with cytochrome, which generally have a threshold partial hydrogen pressure above $10 \mathrm{~Pa}$. This consideration may indicate that products other than acetate were formed by fermentation, leading to less acetate production and thus likely a decrease in availability of acetate for generation of current. In addition, the overall abundance of archaeal 16S rRNA genes was considerably lower in the high-current PMFC than in the low-current PMFC. This result leads to the conclusion that the methanogens likely did not consume a major portion of the acetate available for G. sulfurreducens and G. metallireducens.

De Schamphelaire et al. (2010) performed a phylogenetic community analysis on archaea and found that $45 \%$ of the archaeal clone sequence was not related to any of the known methanogenic lineages. Of the archaeal clone sequences related to methanogenic lineages, $20 \%$ belonged to Methanobacteriaceae, $18 \%$ to Methanosarcina and $10 \%$ to Methanosaetaceae. In this study, the amounts of the acetateutilising methanogens Methanosarcina and Methanosaetaceae were considerably lower (an average of $<10 \%$ versus an average of $28 \%$ ). One possible explanation for this discrepancy is the difference in the availability of graphite as an electron acceptor. De Schamphelaire et al. (2010) used a graphite felt in a sediment as the anode electrode for the PMFC, whereas in our setups we used graphite granules (without sediment) as electrodes. The use of graphite granules may have resulted in the increased availability of

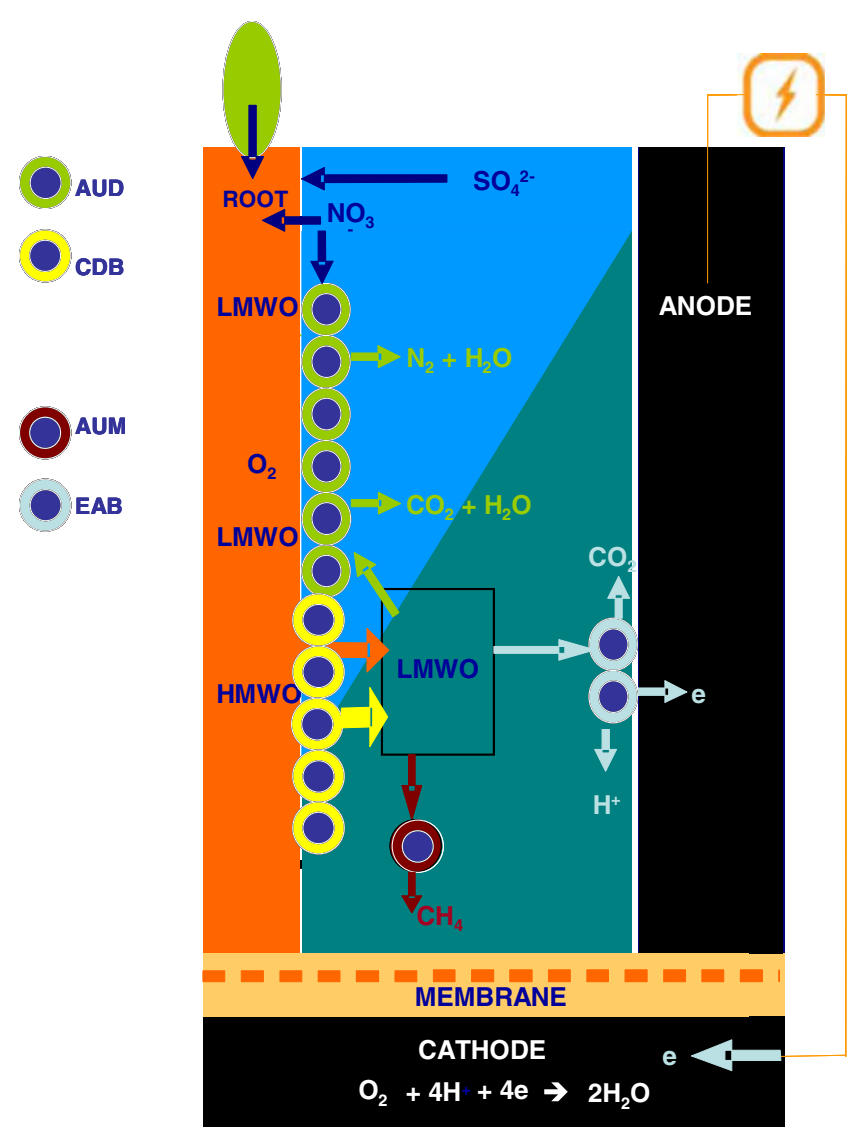

Fig. 5 Schematic presentation of the possible oxidation pathways of high molecular weight organic compounds $(H M W O)$ and low molecular weight organic compounds $(L M W O)$ in the anode of the plant microbial fuel cell (PMFC). The orange arrow represented the LMWO lost by the plant root; the yellow arrow represented the LMWO produced by cellulose-degrading bacteria $(C D B)$. The green, light blue and brown arrows represented the possible oxidation pathways and products of the oxidation of LMWO compounds. The blue colour represents the volume of the PMFC anode where electrochemically active bacteria $(E A B)$ are out-competed by acetate utilising denitrifiers $(A U D)$ for LMWO. The green colour represents the volume of the PMFC anode where EAB are present and thus compete for LMWO with acetate-utilising methanogens (AUM) 
graphite as an electron acceptor. The limited availability of the graphite felt as an electron acceptor may have given the acetate-utilising methanogens a competitive advantage over EAB. In case the availability of graphite as an electron acceptor indeed limits the competitive advantage of EAB over acetate-utilising methanogens, the availability of graphite as an electron acceptor will be an important factor in the reduction of methanogenesis by the PMFC (De Schamphelaire et al. 2008; Strik et al. 2008).

Possible electron sinks and electron donor transfer pathways

Five terminal electron acceptors were present at the anodes of the PMFCs: graphite granules, oxygen, nitrate, sulphate and carbon dioxide. Of these, the latter four are possible electron sinks that do not contribute to current generation. The microbial community at the anode of the PMFC did not contain a large population of sulphatereducing bacteria, indicating that they were out-competed for electron donor by the facultative anaerobic denitrifiers (such as Rhodocyclaceae and Comamonadaceae) and EAB (such as G. sulfurreducens and G. metallireducens) that were present in the anode of the PMFC. This finding is consistent with observations from natural freshwater sediments and wastewater treatment facilities, where the terminal electron acceptors are used in the following order of preference: $\mathrm{O}_{2}, \mathrm{NO}_{3}{ }^{-}, \mathrm{Mn}(\mathrm{IV})$ oxides, $\mathrm{Fe}(\mathrm{III})$ oxides, $\mathrm{SO}_{4}{ }^{2-}$ and $\mathrm{CO}_{2}$. Most $\mathrm{EAB}$, such as $G$. sulfurreducens and $G$. metallireducens, reduce $\mathrm{Fe}(\mathrm{III})$ oxides in natural sediments (Holmes et al. 2004). Iron reducers have a higher rate of conversion of acetate and hydrogen than sulphate-reducing bacteria and methanogens, which gives them a competitive advantage (Van Bodegom and Scholten 2001). Despite the competitive advantage of EAB, however, acetate-utilising methanogens (Methanosarcina) were found. The presence of acetateutilising methanogens might be explained by the availability of $\mathrm{CO}_{2}$ at the root surface, as it is released by the plant roots as the result of metabolic processes. In contrast to $\mathrm{CO}_{2}$, graphite granules have limited availability at the root surface due to the porous structure of the anode, which means that not all root surface was in direct contact with graphite granules. Figure 5 shows a schematic presentation of electron donor pathways and electron sinks.

Anaerobic cellulolytic bacteria dominated the area where most of the EAB were found, and more EAB were found on the graphite granules than on the root surface. This result implies that current was generated via the hydrolysis of cellulose and not directly from low molecular weight rhizodeposits. When optimising electricity generation in a PMFC, therefore, the focus should be on root biomass production and effective hydrolysis of the biomass.
Acknowledgements This research received funding from the European community's 7th framework programme FP7/2007-2013 under grant agreement no. 226532, as well as from Senternovem, the Dutch governmental agency for sustainability and innovation from the Ministry of Finance (grant no. EOSLT06020), and was supported by Alliander. The authors would like to thank Marjolein Helder and Kirsten Steinbusch for their useful comments.

Open Access This article is distributed under the terms of the Creative Commons Attribution License which permits any use, distribution, and reproduction in any medium, provided the original author(s) and the source are credited.

\section{References}

Amann RI, Krumholz L, Stahl DA (1990) Fluorescent-oligonucleotide probing of whole cells for determinative, phylogenetic, and environmental studies in microbiology. J Bacteriol 172:762-770

Amann RI, Zarda B, Stahl DA, Schleifer KH (1992) Identification of individual prokaryotic cells by using enzyme-labeled, rRNA-targeted oligonucleotide probes. Appl Environ Microbiol 58:3007-3011

Bano N, Ruffin S, Ransom B, Hollibaugh JT (2004) Phylogenetic composition of arctic ocean archaeal assemblages and comparison with Antarctic assemblages. Appl Environ Microbiol 70:781-789

Bond DR, Lovley DR (2002) Reduction of Fe(III) oxide by methanogens in the presence and absence of extracellular quinones. Environ Microbiol 4:115-124

Borole AP, Hamilton CY, Aaron DS, Tsouris C (2009) Investigating microbial fuel cell bioanode performance under different cathode conditions. Biotechnol Progr 25:1630-1636

Chaudhuri SK, Lovley DR (2003) Electricity generation by direct oxidation of glucose in mediatorless microbial fuel cells. Nat Biotechnol 21:1229-1232

Coates JD, Bhupathiraju VK, Achenbach LA, McInerney MJ, Lovley DR (2001) Geobacter hydrogenophilus, Geobacter chapellei and Geobacter grbiciae, three new, strictly anaerobic, dissimilatory $\mathrm{Fe}$ (III)-reducers. Int J Syst Evol Microbiol 51:581-588

Collins MD, Lawson PA, Willems A, Cordoba JJ, Fernandez-Garayzabal J, Garcia P, Cai J, Hippe H, Farrow JAE (1994) The phylogeny of the genus Clostridium: proposal of five new genera and eleven new species combinations. Int J Syst Bacteriol 44:812-826

Daims H, Brähl A, Amann R, Schleifer KH, Wagner M (1999) The domain-specific probe EUB338 is insufficient for the detection of all bacteria: development and evaluation of a more comprehensive probe set. Syst Appl Microbiol 22:434-444

De Schamphelaire L, Van Den Bossche L, Hai SD, Hofte M, Boon N, Rabaey K, Verstraete W (2008) Microbial fuel cells generating electricity from rhizodeposits of rice plants. Environ Sci Technol 42:3053-3058

De Schamphelaire L, Cabezas A, Marzorati M, Friedrich MW, Boon N, Verstraete W (2010) Microbial community analysis of anodes from sediment microbial fuel cells powered by rhizodeposits of living rice plants. Appl Environ Microbiol 76:2002-2008

Dekker A, Ter Heijne A, Saakes M, Hamelers HVM, Buisman CJN (2009) Analysis and improvement of a scaled-up and stacked microbial fuel cell. Environ Sci Technol 43:9038-9042

Demaneche S, Sanguin H, Poté J, Navarro E, Bernillon D, Mavingui P, Wildi W, Vogel TM, Simonet P (2008) Antibiotic-resistant soil bacteria in transgenic plant fields. Proc Natl Acad Sci USA 105:3957-3962

Finneran KT, Johnsen CV, Lovley DR (2003) Rhodoferax ferrireducens sp. nov., a psychrotolerant, facultatively anaerobic bacterium that oxidizes acetate with the reduction of Fe(III). Int J Syst Evol Microbiol 53:669-673 
Franks AH, Harmsen HJM, Raangs GC, Jansen GJ, Schut F, Welling GW (1998) Variations of bacterial populations in human feces measured by fluorescent in situ hybridization with group-specific 16S rRNAtargeted oligonucleotide probes. Appl Environ Microbiol 64:33363345

Ginige MP, Keller J, Blackall LL (2005) Investigation of an acetate-fed denitrifying microbial community by stable isotope probing, full-cycle rRNA analysis, and fluorescent in situ hybridization-microautoradiography. Appl Environ Microbiol 71:8683-8691

Griffiths RI, Whiteley AS, O’Donnell AG, Bailey MJ (2000) Rapid method for coextraction of DNA and RNA from natural environments for analysis of ribosomal DNA- and rRNA-based microbial community composition. Appl Environ Microbiol 66:5488-5491

Harmsen HJM, Raangs GC, He T, Degener JE, Welling GW (2002) Extensive set of 16S rRNA-based probes for detection of bacteria in human feces. Appl Environ Microbiol 68:2982-2990

Hartmann A, Schmid M, van Tuinen D, Berg G (2009) Plant-driven selection of microbes. Plant Soil 321:235-257

Helder M, Strik DPBTB, Hamelers HVM, Kuhn AJ, Blok C, Buisman CJN (2010) Concurrent bio-electricity and biomass production in three plant-microbial fuel cells using Spartina anglica, Arundinella anomala and Arundo donax. Bioresource Technol 101:3541-3547

Holmes DE, Bond DR, O'Neil RA, Reimers CE, Tender LR, Lovley DR (2004) Microbial communities associated with electrodes harvesting electricity from a variety of aquatic sediments. Microb Ecol 48:178-190

Hsiao CL, Chang JJ, Wu JH, Chin WC, Wen FS, Huang CC, Chen CC, Lin CY (2009) Clostridium strain co-cultures for biohydrogen production enhancement from condensed molasses fermentation solubles. Int J Hydrogen Energy 34:7173-7181

Ishii S, Hotta Y, Watanabe K (2008) Methanogenesis versus electrogenesis: morphological and phylogenetic comparisons of microbial communities. Biosci Biotechnol Biochem 72:286-294

Jung S, Regan JM (2007) Comparison of anode bacterial communities and performance in microbial fuel cells with different electron donors. Appl Microbiol Biotechnol 77:393-402

Juretschko S, Timmermann G, Schmid M, Schleifer KH, PommereningRöser A, Koops HP, Wagner M (1998) Combined molecular and conventional analyses of nitrifying bacterium diversity in activated sludge: Nitrosococcus mobilis and Nitrospira-like bacteria as dominant populations. Appl Environ Microbiol 64:3042-3051

Kaku N, Yonezawa N, Kodama Y, Watanabe K (2008) Plant/microbe cooperation for electricity generation in a rice paddy field. Appl Microbiol Biotechnol 79:43-49

Kim BH, Kim HJ, Hyun MS, Park DH (1999) Direct electrode reaction of Fe(III)-reducing bacterium, Shewanella putrefaciens. J Microbiol Biotechnol 9:127-131

Lane DJ (1991) 16S/23S rRNA sequencing. In: Stackebrand E, Goodfellow M (eds) Nucleic acid techniques in bacterial systematics, 1st edn. Wiley, New York, pp 115-175

Lovley DR, Kashefi K, Vargas M, Tor JM, Blunt-Harris EL (2000) Reduction of humic substances and Fe(III) by hyperthermophilic microorganisms. Chem Geol 169:289-298

Lynd LR, Wyman CE, Gerngross TU (1999) Biocommodity engineering. Biotechnol Progr 15:777-793

Lynd LR, Weimer PJ, Van Zyl WH, Pretorius IS (2002) Microbial cellulose utilization: fundamentals and biotechnology. Microbiol Mol Biol Rev 66:506-577

Maki M, Leung KT, Qin W (2009) The prospects of cellulaseproducing bacteria for the bioconversion of lignocellulosic biomass. Int J Biol Sci 5:500-516

Manz W, Amann R, Ludwig W, Wagner M, Schleifer K-H (1992) Phylogenetic oligodeoxynucleotide probes for the major subclass of proteobacteria: problems and solutions. Syst Appl Microbiol 15:593-600
Min B, Cheng S, Logan BE (2005) Electricity generation using membrane and salt bridge microbial fuel cells. Water Res 39:16751686

Nicol GW, Glover LA, Prosser JI (2003) Molecular analysis of methanogenic archaeal communities in managed and natural upland pasture soils. Global Change Biol 9:1451-1457

Nielsen JL, Klausen C, Nielsen PH, Burford M, Jørgensen NOG (2006) Detection of activity among uncultured actinobacteria in a drinking water reservoir. FEMS Microbiol Ecol 55:432-438

Niessen J, Schröder U, Scholz F (2004) Exploiting complex carbohydrates for microbial electricity generation - a bacterial fuel cell operating on starch. Electrochem Commun 6:955-958

Offre P, Pivato B, Siblot S, Gamalero E, Corberand T, Lemanceau P, Mougel C (2007) Identification of bacterial groups preferentially associated with mycorrhizal roots of Medicago truncatula. Appl Environ Microbiol 73:913-921

Park DH, Kim BH (2001) Growth properties of the iron-reducing bacteria, Shewanella putrefaciens IR-1 and MR-1 coupling to reduction of $\mathrm{Fe}(\mathrm{III})$ to $\mathrm{Fe}(\mathrm{II})$. J Microbiol 39:273-278

Park HS, Kim BH, Kim HS, Kim HJ, Kim GT, Kim M, Chang IS, Park YK, Chang HI (2001) A novel electrochemically active and Fe (III)-reducing bacterium phylogenetically related to Clostridium butyricum isolated from a microbial fuel cell. Anaerobe 7:297306

Phillips DA, Ferris H, Cook DR, Strong DR (2003) Molecular control points in rhizosphere food webs. Ecology 84:816-826

Pinton R, Varanini Z (2007) The rhizosphere: biochemistry and organic substances at the soil-plant interface. CRC, Boca Raton

Potter MC (1911) Electrical effects accompanying the decomposition of organic compounds. Proc R Soc London, Ser B 84:260-276

Rabaey K, Boon N, Siciliano SD, Verhaege M, Verstraete W (2004) Biofuel cells select for microbial consortia that self-mediate electron transfer. Appl Environ Microbiol 70:5373-5382

Reguera G, Nevin KP, Nicoll JS, Covalla SF, Woodard TL, Lovley DR (2006) Biofilm and nanowire production leads to increased current in Geobacter sulfurreducens fuel cells. Appl Environ Microbiol 72:7345-7348

Ren Z, Ward TE, Regan JM (2007) Electricity production from cellulose in a microbial fuel cell using a defined binary culture. Environ Sci Technol 41:4781-4786

Rezaei F, Xing D, Wagner R, Regan JM, Richard TL, Logan BE (2009) Simultaneous cellulose degradation and electricity production by Enterobacter cloacae in a microbial fuel cell. Appl Environ Microbiol 75:3673-3678

Rismani-Yazdi H, Christy AD, Dehority BA, Morrison M, Yu Z, Tuovinen OH (2007) Electricity generation from cellulose by rumen microorganisms in microbial fuel cells. Biotechnol Bioeng 97:1398-1407

Scheid D, Stubner S, Conrad R (2004) Identification of rice root associated nitrate, sulfate and ferric iron reducing bacteria during root decomposition. FEMS Microbiol Ecol 50:101-110

Schellenberger S, Kolb S, Drake HL (2010) Metabolic responses of novel cellulolytic and saccharolytic agricultural soil bacteria to oxygen. Environ Microbiol 12:845-861

Strik DPBTB, Hamelers HVM, Snel JFH, Buisman CJN (2008) Green electricity production with living plants and bacteria in a fuel cell. Int J Energy Res 32:870-876

Strik DPBTB, Timmers RA, Helder M, Steinbusch KJJ, Hamelers HVM, Buisman CJN (2011) Microbial solar cells: applying photosynthetic and electrochemically active organisms. Trends Biotechnol 29(1):41-49

Strunk O, Ludwig W (1997) ARB: a software environment for sequence data. http://www.mikro.biologie.tu-muenchen.de

Taiz L, Zeiger E (2006) Plant physiology. Sinauer Associates, Sunderland 
Tanner CC (1996) Plants for constructed wetland treatment systems - a comparison of the growth and nutrient uptake of eight emergent species. Ecol Eng 7:59-83

Thauer RK, Kaster AK, Seedorf H, Buckel W, Hedderich R (2008) Methanogenic archaea: ecologically relevant differences in energy conservation. Nat Rev Microbiol 6:579-591

Thomsen TR, Kong Y, Nielsen PH (2007) Ecophysiology of abundant denitrifying bacteria in activated sludge. FEMS Microbiol Ecol 60:370-382

Timmers RA, Strik DPBTB, Hamelers HVM, Buisman CJN (2010) Long-term performance of a plant microbial fuel cell with Spartina anglica. Appl Microbiol Biotechnol 86:973981
Timmers RA, Strik DPBTB, Arampatzoglou C, Buisman CJN, Hamelers HVM (2011) Rhizosphere anode model explains high oxygen levels during operation of a Glyceria maxima PMFC. Bioresource Technol. doi:10.1016/j.biortech.2011.10.088

Van Bodegom PM, Scholten JCM (2001) Microbial processes of $\mathrm{CH}_{4}$ production in a rice paddy soil: model and experimental validation. Geochim Cosmochim Acta 65:2055-2066

Xing D, Zuo Y, Cheng S, Regan JM, Logan BE (2008) Electricity generation by Rhodopseudomonas palustris $D X-1$. Environ Sci Technol 42:4146-4151

Xing D, Cheng S, Logan BE, Regan JM (2010) Isolation of the exoelectrogenic denitrifying bacterium Comamonas denitrificans based on dilution to extinction. Appl Microbiol Biotechnol 85:1575-1587 